\title{
Jacques LE BOHEC, Élections et télévision
}

Grenoble, Presses universitaires de Grenoble, coll. Communication, médias et sociétés, 2007, 208 p.

\section{Marieke Stein}

\section{(C) OpenEdition}

\section{Journals}

Édition électronique

URL : http://journals.openedition.org/questionsdecommunication/1915

DOI : 10.4000/questionsdecommunication. 1915

ISSN : 2259-8901

\section{Éditeur}

Presses universitaires de Lorraine

\section{Édition imprimée}

Date de publication : 1 juillet 2008

Pagination : 378-381

ISBN : 978-2-86480-952-4

ISSN : 1633-5961

\section{Référence électronique}

Marieke Stein, « Jacques Le вонеc, Élections et télévision », Questions de communication [En ligne], 13 | 2008, mis en ligne le 01 juillet 2010, consulté le 08 avril 2021. URL : http://journals.openedition.org/ questionsdecommunication/1915; DOI : https://doi.org/10.4000/questionsdecommunication.1915

Ce document a été généré automatiquement le 8 avril 2021.

Tous droits réservés 


\title{
Jacques LE BOHEC, Élections et télévision
}

\author{
Grenoble, Presses universitaires de Grenoble, coll. Communication, \\ médias et sociétés, 2007, 208 p.
}

\section{Marieke Stein}

\section{RÉFÉRENCE}

Jacques LE BонЕс, Élections et télévision. Grenoble, Presses universitaires de Grenoble, coll. Communication, médias et sociétés, 2007, 208 p.

1 Les élections de 2002, puis celles de 2007, ont ravivé un débat qui occupe depuis plusieurs années les politologues et les spécialistes des médias : quelle est l'influence de la télévision sur les votes des électeurs? Contre les théories selon lesquelles les médias influencent les choix politiques des citoyens, Jacques Le Bohec, se penchant sur l'impact des campagnes politiques à la télévision, entend minimiser cet impact lors des élections, en considérant à la fois le rôle des médias et «les diverses contraintes sociales et mentales ainsi que les étapes successives des processus sociaux mis à l'œuvre» (p. 12). Il appuie ses analyses sur les acquis des sciences sociales (histoire, sociologie, sciences de l'information et de la communication, science politique, ethnologie et anthropologie), en mobilisant de nombreux travaux à l'appui de sa thèse, selon laquelle les médias ne déterminent pas les choix des électeurs, les campagnes médiatiques pouvant même aboutir à l'effet inverse de celui qui est recherché. Pour étayer cette thèse, l'auteur commence par interroger la notion même de " pouvoir des médias", puis se penche sur les relations entre les journalistes et les hommes politiques, sur la politique à la télévision et sur les campagnes préélectorales, avant de s'interroger sur la signification du vote. La dernière partie de l'étude, sans doute la plus originale, est consacrée à la soirée électorale télévisée, à ses objectifs et ses significations.

2 Rejetant l'expression magique " pouvoir des médias » au profit de l'idée qu'il existe des interactions variées et fluctuantes entre acteurs politiques et médias, Jacques Le Bohec interroge d'abord les rapports presse/politique, préférant parler de «besoin 
réciproque » des protagonistes, les médias ayant besoin d'informations livrées par les acteurs politiques et les acteurs politiques ayant besoin d'une publicité que seuls les médias peuvent leur donner. Les rapports de force entre le champ politique et le champ médiatique sont donc variables, et chacun dispose de moyens de gratifier ou de sanctionner l'autre (du côté des politiques, boycott d'un journaliste, rétention d'information...; du côté des journalistes, interviews agressives ou silence total...). Toute cette partie de la démonstration a pour but de montrer que, même s'ils cherchent à instrumentaliser les médias, les acteurs politiques ne sont pas toujours en mesure de leur imposer leurs vues.

3 Le chapitre "La politique à la télévision" propose un historique des contraintes officielles sur ce média, montrant que les journalistes ont aujourd'hui le sentiment d'être entièrement libres, en l'absence de contraintes formelles comme celles qui ont pu exister sous I'ORTF et le contrôle gouvernemental direct. En réalité, explique l'auteur, les gouvernants ont d'autres moyens de se concilier les médias: nomination de directeurs de chaîne favorables au gouvernement, mode d'élection des membres du CSA, pressions sur les journalistes, et même censure, comme l'illustre le cas d'un reportage jugé subversif de Pierre Caries, "Pas vu pas pris » (1995), à propos des connivences entre politiques et journalistes, qui fut censuré en 1995 sur Canal+ et ne fut jamais diffusé. L'auteur évoque aussi le fait que les journalistes peuvent soutenir discrètement les gouvernements par des reportages orientés ou l'évitement de certains sujets, et rappelle les propos de Roland Barthes (Mythologies, Paris, Éd. Le Seuil, 1957) sur le fait que les journaux télévisés diffusent une idéologie implicite.

La seconde moitié de l'ouvrage aborde plus précisément le rôle de la télévision lors des campagnes électorales, où les journalistes se mobilisent au même titre que d'autres champs professionnels: c'est la "mobilisation multisectorielle». Les actions de communication des politiques à l'endroit des médias sont énumérées, depuis la fabrication d'événements et de "petites phrases" jusqu'à la sélection, pour les interviews, de journalistes complaisants.Tout en se refusant toujours à leur reconnaître un « effet persuasif» sur les votes des électeurs, Jacques Le Bohec reconnaît que « les médias ne disent pas quoi voter mais à quoi penser quand l'on vote» (p. 122), et énumère une série de procédés propres à orienter les choix des spectateurs, comme les effets de cadrage, qui consistent à sélectionner certains aspects de la réalité en fonction du jour sous lequel on veut la montrer En définitive, l'auteur reconnaît surtout aux médias un rôle essentiel dans la mobilisation des électeurs. Cela apparaît en particulier dans le chapitre consacré à la définition du vote et à sa signification sociale, qui l'apparente à un rituel. Ce n'est pas tant la description de ce rituel qui importe que la signification que lui donne l'auteur qui est de "consacre[r] une division du travail politique entre gouvernants d'un côté (sacré) et gouvernés de l'autre (profanes)» (p. 136). Suit une analyse sociologique des habitudes électorales qui dépendent de la situation sociale des électeurs, de leur origine sociale et géographique, de leur degré d'intérêt pour la politique. Cependant, on peut s'interroger sur la validité de la conclusion qui considère que les campagnes de communication n'influent pas sur les votes, au contraire des déterminismes sociaux et mentaux. Certes, ceux-ci sont fondamentaux. Mais comment expliquer qu'en 2007, une partie de l'électorat «traditionnel » du FN ait opté pour Nicolas Sarkozy ou que des socialistes de longue date aient été séduits par François Bayrou, si l'on n'envisage pas le rôle des médias? 
Celui-ci s'est peut-être renforcé lors des dernières élections, mais l'absence de ces questions dans l'étude de Jacques Le Bohec affaiblit certaines de ses affirmations.

5 il donne de nombreux contre-exemples, comme le cas de Jacques Chirac qui, en 2002, «a remonté la pente de la popularité en partie grâce à l'aide involontaire des Guignols de l'Info » (p. 15). Il explique aussi que les sondages ont plus d'impact que les médias dans les choix des électeurs; or, qui diffuse les résultats des sondages, sinon les médias? De même, s'il est indéniable, comme l'affirme l'auteur que le citoyen est influencé avant tout par des structures sociales et mentales et par la pression de son groupe social, n'aurait-il pas fallu s'interroger sur la part des médias dans la formation des opinions de ces groupes? Enfin, lors d'un historique des différents modèles expliquant l'influence de la télévision (du modèle techniciste de l'après-guerre à la cybernétique et à l'école de Francfort), l'auteur réfute tout effet persuasif, même indirect, des messages médiatiques et des discours politiques, insinuant que ceux-ci n'influencent pas les électeurs. Mais alors, à quoi servent-ils? En bref, on peut douter que l'influence de la télévision ne soit qu'une invention de journalistes intéressés à présenter leur activité (et leur entreprise) comme essentielles à la réussite électorale des candidats, et l'on peut se demander pourquoi, si l'influence de la télévision est si faible, la première guerre du Golfe, par exemple, a donné lieu à une telle campagne de désinformation sur les chaînes américaines.

dépit de la qualité des sources mobilisées dans son ouvrage, Jacques Le Bohec ne parvient donc pas réellement à convaincre son lecteur de l'innocuité des médias en matière politique. D'abord, parce que, dans le premier chapitre, il semble vouloir réduire leur impact à un stimulus pavlovien (théorie de Vance Packard, La persuasion clandestine, trad. de l'américain par Hélène Claireau, Paris, Calmann-Lévy [1957] 1958 ; et de Jean-Marie Domenach, La propagande politique, Paris, Presses universitaires de France, 1965), quitte à simplifier parfois la pensée d'autres auteurs. Ensuite, tout en répétant que la télévision ne détermine pas le vote,

7 Évidemment, beaucoup d'autres analyses de Jacques Le Bohec sont très pertinentes, comme lorsqu'il rappelle que les électeurs consomment des médias en phase avec leur grille de lecture du politique, ou qu'un message médiatique n'est jamais univoque. Mais il est dommage qu'elles servent à nier la notion d'" effets " alors qu'elles pourraient conduire à une investigation approfondie sur le rôle de la télévision dans la formation de l'opinion. Il semble également que la campagne présidentielle de 2007 a modifié et accentué très nettement le rôle des médias (et en particulier de la télévision) dans les élections, et qu'elle condamne quelques pages du livre de Jacques Le Bohec, pourtant très récent, à une certaine obsolescence, surtout lorsque l'auteur appuie certaines de ses analyses sur des études elles-mêmes datées, en convoquant, par exemple, The Peoples Choice, de Paul Lazarsfeld, daté de 1944, pour affirmer que seule une petite partie de la population (masculine, aisée, instruite) est touchée par le discours médiatique.

La force de l'ouvrage est surtout dans la description du monde journalistique, spécialité de Jacques Le Bohec qui n'oublie jamais d'envisager aussi les médias comme des entreprises et l'information comme un marché ; Élections et télévision propose également un panorama intéressant des études existantes sur les rapports entre la politique et les médias. À cet égard, l'ouvrage s'apparente souvent à une série de synthèses sur différents thèmes (histoire des émissions politiques, techniques de la communication politique, histoire et sociologie des élections.). Le dernier chapitre, consacré aux soirées électorales télévisées et à leur double dimension commerciale et politique, est le plus 
original et le plus intéressant. Là encore, l'historique (depuis l'instauration progressive de ces soirées dans les années 1965-1974) et la description du grand cérémonial télévisé importent moins que certaines interprétations qui sont données de ce rituel cathodique. L'une des fonctions essentielles de la soirée électorale est de donner une signification univoque à des votes qui obéissent en réalité à des logiques multiples. Quant à la clôture de cette soirée, avec ses " remerciements » adressés par les acteurs politiques aux électeurs, sa signification est l'une des idées fortes de l'ouvrage: en "remerciant» leurs électeurs, les vainqueurs consacrent le retour à une séparation entre le champ politique et les citoyens " profanes ", et à un système représentatif où le citoyen, après la brève mobilisation électorale, délègue une nouvelle fois ses pouvoirs aux acteurs politiques. Ce rôle de "resectorisation» du rituel télévisé est, selon l'auteur plus signifiant que de prétendus « effets persuasifs » difficiles à cerner de la télévision sur les électeurs. Enfin, ces soirées consacrent la domination provisoire du média sur les politiques, dans la mesure où ce soir-là, les journalistes désignent " officiellement» le vainqueur, adoubent les acteurs politiques, et accompagnent le rituel du vote en lui octroyant «une plus-value sociale et symbolique » (p. 185). Ce dernier chapitre ouvre la réflexion, bien au-delà de la seule période électorale, sur le rôle des médias dans la démocratie, selon un angle rarement exploré.

\section{AUTEURS}

\section{MARIEKE STEIN}

CREM, université Paul Verlaine-Metz,marieke.stein@wanadoo.fr 